\title{
Health professional's perceptions of and potential barriers to smoking cessation care: a survey study at a dental school hospital in Japan
}

\author{
Atsushi Saito ${ }^{1 *}$, Makiko Nishina ${ }^{2}$, Keiko Murai ${ }^{3}$, Akiko Mizuno ${ }^{4}$, Fumie Ueshima ${ }^{4}$, Takemi Makiishi ${ }^{1}$, \\ Tatsuya Ichinohe $e^{1,5}$
}

\begin{abstract}
Background: Smoking is currently accepted as a well-established risk factor for many oral diseases such as oral cancer and periodontal disease. Provision of smoking cessation care to patients with oral problems is a responsibility of health care professionals, particularly dentists and dental hygienists. This study examined the smoking-related perceptions and practices of dental school hospital-based health professionals in Japan.

Findings: A cross-sectional study design was used. The sample was formed from dentists, dental hygienists, physicians and nurses of a dental school hospital in Tokyo, Japan $(n=93,72 \%)$. Participants were asked to complete an 11-item questionnaire assessing demographic variables and smoking history, provision of smoking cessation advice or care, attitudes about smoking cessation, and perceived barrier(s) to smoking cessation care. Eighteen percent of participants reported being current smokers and 15\% reported being ex-smokers, with higher smoking rates reported by dentists compared with other health professionals $(p=0.0199)$. While recognizing the importance of asking patients about their smoking status, actual provision of smoking cessation advice or care by participants was relatively insufficient. Interventions such as 'assess willingness to make a quit attempt' and 'assist in quit attempt' were implemented for less than one-quarter of their patients who smoke. Non-smokers were more likely to acknowledge the need for increased provision in smoking cessation care by oral health professionals. 'Lack of knowledge and training' was identified as a central barrier to smoking cessation care, followed by 'few patients willing to quit'.

Conclusions: A need for further promotion of smoking cessation activities by the health professionals was identified. The findings also suggest that dentists and dental hygienists, while perceiving a role in smoking care, do require training in the provision of smoking cessation care to hospital patients. In order to overcome the potential barriers, it is necessary to provide staff with appropriate training and create an atmosphere supportive of smoking cessation activities.
\end{abstract}

\section{Background}

Tobacco use is one of the leading causes of preventable mortality in industrialized nations [1]. In 2002, World Health Organization [2] reported that about one in eight deaths in Japan are due to smoking (about 100,000 deaths a year), and Japan had some of the most lenient anti-tobacco laws among developed nations. Since then, considerable efforts have been made to promote

\footnotetext{
* Correspondence: atsaito@tdc.ac.jp

'Department of Clinical Oral Health Science, Tokyo Dental College, 2-9-18

Misaki-cho, Chiyoda-ku, Tokyo, 101-0061, Japan

Full list of author information is available at the end of the article
}

smoking cessation activities. The national project, 'Health Japan 21' includes tobacco control, and the prevention of passive smoking was included in the Health Promotion Law implemented in 2003 [3]. Furthermore, in 2006, smoking cessation treatment by physicians was approved under the national health care insurance in Japan via the recognition of 'nicotine dependence' as a disease [4]. However, in 2008, it was shown that $36.8 \%$ of Japanese adult men and $9.1 \%$ of women still smoke regularly [5]. Given this situations, it is important for health professionals in Japan to further promote smoking cessation program.

\section{Biomed Central}


Smoking is accepted as a well-established risk factor for many oral diseases including oral cancer and periodontal disease [6]. Oral health professionals are in a unique position to advise smokers to quit by providing effective counseling on the various aspects of tobaccoinduced diseases. Dentists and dental hygienists have regular contact with smokers and a great potential for helping their patients to quit smoking; yet, this potential is often underutilized [7-14]. It has also been suggested that Japanese health professionals may lack sufficient awareness of their position as role models for dental patients, in comparison to western developed countries $[15,16]$.

Concurrently with the inclusion of smoking cessation intervention by physicians into the national health care insurance plan in Japan, a smoking cessation program was initiated at Suidobashi Hospital, Tokyo Dental College, in 2006. The program is a new multi-disciplinary approach to smoking cessation, which includes interventions by the medical and dental professionals. While the program has been a significant addition to our patient care [17], it was our general feeling that more effort was needed to implement smoking cessation care to patients receiving dental treatments. As part of an effort to further promote smoking cessation activities, this study assessed smoking-related perceptions, attitudes and practices of dental school hospital-based health professionals.

\section{Methods}

A cross-sectional survey of health professionals was conducted at Suidobashi Hospital, Tokyo Dental College, Tokyo, Japan in July 2010. Among the practicing professionals, those who were providing direct care to patients were asked to participate in the study.

\section{Questionnaire}

A novel questionnaire was developed in order to assess smoking-related perceptions, attitudes and practices of health professionals. Specifically, the basic structure of the questionnaire items was generated in reference to the published studies $[13,18]$ and to the Clinical Practice Guideline by U.S. Department of Health and Human Services [19]. Where required, one dentist, fluent in both Japanese and English performed the forward translation. A survey study in Japan [18] was also used as a guide for translation, as some of the wording in the questionnaire was similar to the material being translated. The base questionnaire was piloted with a small group of health professionals (two dentists, one dental hygienist, one nurse and one physician) in order to ensure the clarity and comprehensiveness of the questionnaire. The final and refined version (See additional file 1: Items in the survey questionnaire) was composed of the following 11 items. In Items 1 to 4 , participant demographics were assessed. In Item 5, smoking status and history were requested. Items 6 and 7 inquired about participants' experiences with smoking cessation care training and their willingness to receive it. In Item 8 , the provision of smoking assessment or cessation care to patients was assessed, with four subscale items on a 10-point scale, asking the participants how often they performed the intervention specified from never $(0 \%)$ to always (100\%). Where appropriate, the answers were dichotomised; the cut-off point was a median score of $50 \%$. In Item 9, we assessed participants' perceptions regarding smoking or smoking cessation care, with seven subscale items on a 5-point scale, asking if they strongly disagree (1) or agree (5) with a given statement. Where appropriate, the answers were dichotomized ('strongly disagree', 'somewhat disagree', 'not sure either way' versus 'somewhat agree' and 'strongly agree'). In Item 10, the participants' potential barriers were assessed. In the final Item 11, the participants were asked to express their views on smoking or smoking cessation care.

\section{Ethical considerations}

Approval for the study protocol and the survey implementation was obtained from the Suidobashi Hospital's review board. A formal ethics review was waived by the institutional review board, because the research involved submitting a questionnaire to adults who complied of their own free will and, any information that would identify participants was avoided. Written informed consent was obtained from all participants.

\section{Statistical analysis}

All the data obtained was examined and the responses were coded. The data was then descriptively analyzed and an appropriate test was applied, using a statistical package, InStat 3.1 (GraphPad, La Jolla, CA). Chisquared test and one-way analysis of variance (ANOVA) with Tukey-Kramer multiple comparisons test were used.

A p value of less than 0.05 was considered as statistically significant.

\section{Results}

\section{Characteristics of study participants}

In total, 129 questionnaires were distributed, of which 105 were returned. Of these 93 (72\%) were fully completed responses from practicing professionals.

Demographic data were listed in Table 1. The participants comprised predominantly oral health professionals; $58 \%$ were dentists and $24 \%$ were dental hygienists. $16 \%$ were registered nurses. Only small number of physicians $(n=2)$ participated, mainly because of 
Table 1 Demographic characteristics and smoking status of the sample

\begin{tabular}{|c|c|c|c|c|}
\hline & Overall $(n=93)$ & Dentist $(n=54)$ & Dental hygienist $(n=22)$ & Medical $(n=17)$ \\
\hline \multicolumn{5}{|l|}{ Gender } \\
\hline Male & $35(37.6)$ & $35(64.8)$ & $0(0)$ & $0(0)$ \\
\hline Female & $58(62.4)$ & $19(35.2)$ & $22(100)$ & $17(100)$ \\
\hline \multicolumn{5}{|l|}{ Age (in years) } \\
\hline $20-29$ & $30(32.3)$ & $20(37.0)$ & $10(45.5)$ & $0(0)$ \\
\hline $30-39$ & $33(35.5)$ & $20(37.0)$ & $4(18.2)$ & $9(52.9)$ \\
\hline $40-49$ & $15(16.1)$ & $5(9.3)$ & $6(27.3)$ & $4(23.5)$ \\
\hline $50-59$ & $12(12.9)$ & $8(14.8)$ & $1(4.5)$ & $3(17.6)$ \\
\hline $60 \leq$ & $3(3.3)$ & $1(1.9)$ & $1(4.5)$ & $1(5.9)$ \\
\hline \multicolumn{5}{|c|}{ Professional experience (in years) } \\
\hline$<2$ & $16(17.0)$ & $12(22.2)$ & $4(18.2)$ & $0(0)$ \\
\hline $2-4$ & $15(16.1)$ & $12(22.2)$ & $3(13.6)$ & $0(0)$ \\
\hline $5-9$ & $21(22.6)$ & $14(25.9)$ & $4(18.2)$ & $3(17.6)$ \\
\hline $10-19$ & $16(17.0)$ & $4(7.4)$ & $3(13.6)$ & $9(52.9)$ \\
\hline $20 \leq$ & 25 (26.9) & $12(22.2)$ & $8(36.4)$ & $5(29.4)$ \\
\hline \multicolumn{5}{|l|}{ Smoking } \\
\hline Current & $17(18.3)$ & 15 (27.7) & $2(9.1)$ & $0(0)$ \\
\hline Previous & $14(15.0)$ & $10(18.5)$ & $4(18.2)$ & $0(0)$ \\
\hline Never & $62(66.7)$ & $29(53.7)$ & $16(72.7)$ & 17 (100) \\
\hline
\end{tabular}

Data were shown as number (\%)

the dental school-based hospital setting. Therefore, data for nurses and physicians were pooled to represent medical professionals for the subsequent analysis.

Overall, nearly two-thirds (62\%) of participants were women. About $65 \%$ of dentists were men, but all other professionals comprised women. About two-thirds of participants were aged 20-39 years. There was wide variety in the number of years after receiving professional qualifications. All medical professionals had at least 5 years of practicing experience, while $41 \%$ of dental professionals had fewer than 4 years of experience.

Altogether, $18 \%$ of participants reported being current smokers (17\% of men and 19\% of women), $15 \%$ reported being ex-smokers and $67 \%$ reported never smoking. Higher smoking rates were reported by dentists compared with other professionals ( $\mathrm{p}=0.0199$, Chi-squared test for independence). None of the physicians or nurses who participated in the study was current smoker.

\section{Past training in smoking cessation care}

The majority of participants reported that they have had never received training in smoking cessation care. Only $8 \%$ had previous training, and $71 \%$ indicated that they would like to receive it.

\section{Smoking assessment or cessation care}

We next assessed the provision of smoking assessment or cessation care to patients. Among the four components of a brief intervention, 'asking smoking status' was the most frequently performed activity, followed by 'advise to quit'. (Table 2). Dentists and medical professionals asked about smoking status significantly more than dental hygienists $(\mathrm{p}<0.05, \mathrm{p}<0.01$, respectively; one-way ANOVA with Tukey-Kramer multiple comparisons test). Medical professionals were more likely to advise their patients to quit smoking than dental hygienists $(\mathrm{p}<0.05)$.

'Assess willingness to make a quit attempt' and 'assist in quit attempt' were the interventions implemented for less than one-quarter of their patients. There were no statistically significant differences in any of those interventions among the health professionals. There were no significant differences in provision of smoking assessment or cessation care between smokers and nonsmokers, as assessed by Chi-squared test.

\section{Perception of smoking and smoking cessation}

The participants' perceptions regarding smoking or smoking cessation care were assessed (Table 3). There was a statistically significant difference among overall scores $(\mathrm{p}<0.0001)$. 'It is important to ask smoking status' and 'oral health professionals should participate more in smoking cessation care' were the subscale items with relatively high scores.

There were no significant differences in these perceptions between professional types. When the relationship with smoking status was assessed, a statistically significant difference was noted in responses to 'oral health professionals should participate more in smoking cessation care' ( $\mathrm{p}=0.0404$, Chi-squared test). Non-smokers were more likely to agree with this notion than smokers. 
Table 2 Provision of smoking assessment or cessation care

\begin{tabular}{lllll}
\hline & Overall $(\mathbf{n}=\mathbf{9 3})$ & Dentist $(\mathbf{n}=\mathbf{5 4})$ & Dental hygienist $(\mathbf{n}=\mathbf{2 2})$ & Medical $(\mathbf{n}=\mathbf{1 7})$ \\
\hline Asking smoking status & $66.9(36.2)$ & $68.5(36.3)^{*}$ & $46.4(32.3)$ & $88.2(27.2)^{* *}$ \\
Advise to quit & $43.9(36.5)$ & $43.3(36.4)$ & $30.9(25.6)$ & $62.4(42.8)^{*}$ \\
Assess willingness to make a quit attempt & $22.5(28.3)$ & $23.3(28.9)$ & $22.2(23.1)$ & $20.0(33.5)$ \\
Assist in quit attempt & $17.3(25.2)$ & $14.6(21.0)$ & $19.1(21.6)$ & $23.5(39.0)$ \\
\hline
\end{tabular}

Data were shown as mean \% (SD)

${ }^{*} p<0.05,{ }^{* *} p<0.001$, significantly different from dental hygienist; one-way analysis of variance (ANOVA) with Tukey-Kramer multiple comparisons test

\section{Perceived barriers to smoking cessation care}

'Lack of knowledge and training' (61\%) was identified as a central barrier to smoking cessation care, followed by 'few patients willing to quit' (45\%) (Figure 1). No statistically significant associations were noted between the demographic aspects including years in practice and the barriers that limit the health care professionals from providing smoking cessation care.

While many of the individual comments in Item 11 were related to the perceived barriers expressed in the previous item, they also reflected a general positive attitude toward provision of smoking cessation care.

\section{Discussion}

This study aimed to identify smoking-related perceptions and practices of health care professionals at a dental-school based hospital in Japan. Despite a general positive perception towards smoking cessation, the provision as well as the extent of smoking cessation care by the study participants was found to be relatively low. The findings of the present study support the notion that potential of oral health professionals in smoking cessation is underutilized [7-14].

Oral health professionals have a role in encouraging their patients to quit smoking and helping them, via referral pathways. Also, dental staff should be trained and given the resources to deliver the advice themselves [20]. By the mid 1990's, a quarter of tobacco users in the United States had been advised to quit by their dentist [21]. However, even in northern Europe and North America, a substantial proportion of dental professionals either do not provide smoking cessation interventions or provide them on a very limited basis [22,23]. Little information is available on smoking cessation practices of oral health professionals in Asia. In a survey study of 152 dentists practicing in Malaysia [24], only $17.9 \%$ of them were actually involved in smoking cessation counselling. Consistent with these reports, the dentists and dental hygienists in the present study indicated that smoking cessation interventions such as 'assess willingness to make a quit attempt' and 'assist in quit attempt' were implemented for less than one-quarter of their patients who smoke. The efficacies of smoking-cessation intervention in dental or medical-dental settings were shown in Japan [4,17], suggesting the feasibility of integrating dentists and dental hygienists in a medical smoking cessation intervention. Encouragingly, in a recent survey study of 435 certified periodontists of the Japanese Society of Periodontology, a relatively high percentage $(54.7 \%)$ of the participants indicated that they provide some form of smoking cessation care to their patients [25]. Initiatives are needed to further promote smoking cessation care in our practice setting.

Smoking behaviour among health professionals can be a significant obstacle in promoting smoking cessation initiatives [13,22]. A survey conducted among members of the Japan Medical Association in 2008 showed that the prevalence of cigarette smoking

Table 3 Perception of smoking and smoking cessation

\begin{tabular}{|c|c|c|c|c|}
\hline & $\begin{array}{l}\text { Overall }(n= \\
93)\end{array}$ & $\begin{array}{l}\text { Dentist }(n= \\
54)\end{array}$ & $\begin{array}{l}\text { Dental hygienist ( } \mathrm{n} \\
\quad=22 \text { ) }\end{array}$ & $\begin{array}{l}\text { Medical }(n= \\
17)\end{array}$ \\
\hline Q9-1 We should set an example by not smoking & $3.9(1.2)^{*}$ & $3.7(1.3)$ & $4.2(0.7)$ & $4.0(1.0)$ \\
\hline Q9-2 It is important to ask smoking status & $4.6(0.7)^{*}$ & $4.6(0.8)$ & $4.5(0.5)$ & $4.7(0.5)$ \\
\hline Q9-3 Most patients would not quit smoking anyway & $3.1(0.9)^{*}$ & $3.2(0.8)$ & $3.1(0.9)$ & $2.8(1.1)$ \\
\hline $\begin{array}{l}\text { Q9-4 It is not easy to quit smoking because many smokers are addicted to } \\
\text { nicotine }\end{array}$ & $4.1(0.9)^{*}$ & $4.1(0.9)$ & $4.2(0.8)$ & $4.0(1.1)$ \\
\hline $\begin{array}{l}\text { Q9-5 Oral health professionals should participate more in smoking cessation } \\
\text { care }\end{array}$ & $4.1(0.9)^{*}$ & $4.0(0.9)$ & $4.0(0.7)$ & $4.4(0.7)$ \\
\hline Q9-6 Oral health professionals' time would be better spent on other activities & $2.6(1.0)^{*}$ & $2.8(1.0)$ & $2.4(0.8)$ & $2.5(1.2)$ \\
\hline $\begin{array}{l}\text { Q9-7 Dental patients have other important needs, so they have no time for } \\
\text { smoking cessation care }\end{array}$ & $2.3(0.9)^{*}$ & $2.4(0.9)$ & $2.2(0.7)$ & $2.1(1.0)$ \\
\hline
\end{tabular}

Data are shown as mean score (SD) from the rating scale of 1-5. A higher score indicates greater agreement with the statement.

*Significantly different between subscale items, $p<0.0001$, ANOVA with Tukey-Kramer multiple comparisons test 


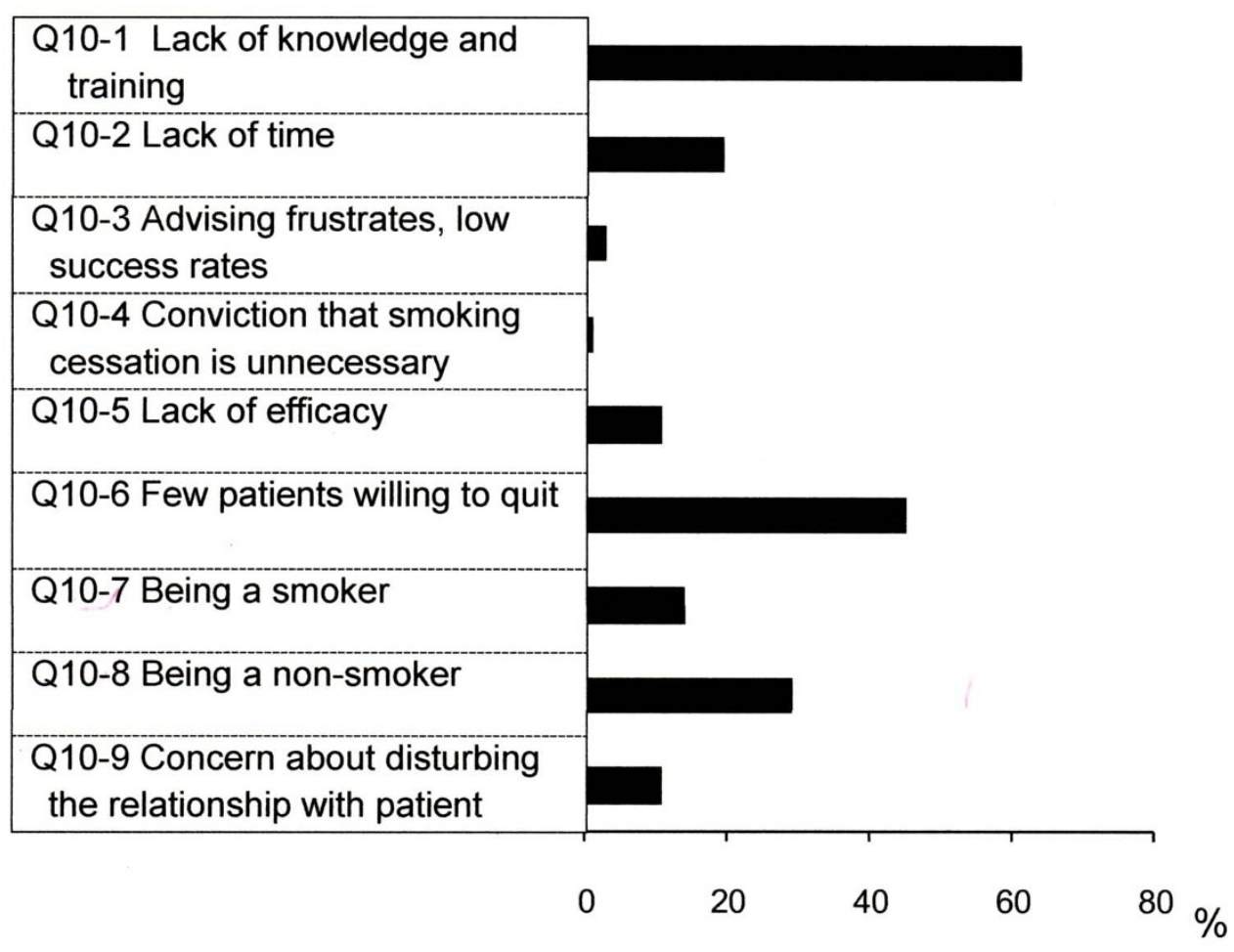

Figure 1 Perceived barriers to smoking cessation care. Data were shown as \% response (the sum of percentages exceeds $100 \%$ because multiple answers were allowed)

among 3,486 Japanese physicians was $15.0 \%$ for men and $4.6 \%$ for women [26]. A recent survey study conducted among 1000 dentists in Japan revealed that the prevalence of regular smokers was $25 \%$ for men and $3 \%$ for women [27]. In the present study, prevalence of smokers among women (19\%) was relatively higher than that reported for the general population [5] or for Japanese dentists in private practice [27]. However, this needs to be interpreted with caution, since nearly two-thirds of the participants were women. The overall prevalence of smokers was higher than that (14.7\%) reported for the certified periodontists [25]. Tobaccousing health professionals, including dentists, were reported to be less proactive than their non-using counterparts [15,28-30]. This trend was supported by our finding that non-smokers were more likely to feel that oral health professionals should participate more in smoking cessation care, when compared to smokers. These results suggested that further effort in smoking cessation among the health professionals in our hospital is needed.
We found that interventions by the dental hygienists in smoking cessation care were somewhat limited when compared to other health professionals (Table 2). This may be due to the fact that the dental hygienists working in our hospital have not been formally integrated into the smoking cessation treatment program. Given the importance of the role of oral health personnel in smoking prevention and control, an active participation of dental hygienists in the smoking cessation treatment program is necessary.

In the present study, lack of training and knowledge was considered to be the major barrier that potentially limited the involvement of the health professionals in smoking cessation activities. Also, more than one third of the participants perceived that few patients would be willing to quit. Removing such barriers can be difficult [31]. A Cochrane Review [32] on the training of health professionals in smoking cessation concluded that health professionals who were trained were better at delivering smoking cessation interventions. In the present study, no statistically significant associations were noted 
between the barriers and the demographic aspects including years in practice. Given that only $8 \%$ of the participants had prior training in smoking cessation care, it is necessary to offer opportunities for adequate training.

Rosseel et al. [13] reported that an atmosphere supportive of advice among colleagues is important in the implementation of strategies which support smoking cessation. Support from our own practice members and organization may increase the efficacy of the health professionals in smoking cessation care.

This study has several inherent limitations. The data were collected via a self-reported questionnaire. Like all questionnaires, the possibility of both intentional and unintentional mis-reporting threatens the validity and reliability of the findings. Relatively small number of participants allowed limited statistical analysis of data.

However, the present study adds to the existing literature by providing the salient information regarding the perceptions of and potential barriers to smoking cessation care among multiple health professions in Japan.

\section{Conclusions}

We identified a need for further promotion of smoking cessation activities by the hospital-based health professionals. Hospital policies and faculty education providers need to provide staff with appropriate training and create an atmosphere supportive of smoking cessation activities.

\section{Consent}

Written informed consent was obtained from the participants for publication of this manuscript and accompanying materials. A copy of the written consent is available for review by the Editor-in-Chief of this journal.

\section{Additional material}

Additional file 1: The items in the survey questionnaire

\begin{abstract}
Author details
'Department of Clinical Oral Health Science, Tokyo Dental College, 2-9-18 Misaki-cho, Chiyoda-ku, Tokyo, 101-0061, Japan. ${ }^{2}$ Department of Internal Medicine, Tokyo Dental College, 2-9-18 Misaki-cho, Chiyoda-ku, Tokyo, 1010061, Japan. ${ }^{3}$ Section of Nursing, Tokyo Dental College Suidobashi Hospital, 2-9-18 Misaki-cho, Chiyoda-ku, Tokyo, 101-0061, Japan. ${ }^{4}$ Section of Dental Hygiene, Tokyo Dental College Suidobashi Hospital, 2-9-18 Misaki-cho, Chiyoda-ku, Tokyo, 101-0061, Japan. ${ }^{5}$ Department of Dental Anesthesiology, Tokyo Dental College, 1-2-2 Masago, Mihama-ku, Chiba, 261-8502, Japan.
\end{abstract}

\section{Authors' contributions}

AS designed the study, constructed the survey questionnaire, performed data analyses, and drafted the manuscript. AM, FU and MK contributed to data collection and analysis. MN interpreted the study and edited the manuscript. TM and TI oversaw procedures. All authors have read and approved the manuscript.

\section{Competing interests}

The authors declare that they have no competing interests.

Received: 27 August 2010 Accepted: 7 December 2010

Published: 7 December 2010

\section{References}

1. Rigotti NA: Clinical practice. Treatment of tobacco use and dependence. N Eng J Med 2002, 346:506-12.

2. World Health Organization: WHO fact sheets, Smoking statistics. 2002 [http://www.wpro.who.int/media_centre/fact_sheets/fs_20020528.htm].

3. Udagawa K, Miyoshi M, Yoshiike N: Mid-term evaluation of "Health Japan 21": focus area for the nutrition and diet. Asia Pac J Clin Nutr 2008, 17(S2):445-452.

4. Hanioka T, Ojima M, Tanaka H, Naito M, Hamajima N, Matsuse R: Intensive smoking-cessation intervention in the dental setting. J Dent Res 2010, 89:66-70.

5. Ministry of Health Labour and Welfare: National nutrition survey in 2008. [http://www.mhlw.go.jp/houdou/2008/12/dl/h1225-5d.pdf], in Japanese.

6. Hilgers KK, Kinane DF: Smoking, periodontal disease and the role of the dental profession. Int J Dent Hyg 2004, 2:56-63.

7. Tomar SL: Dentistry's role in tobacco control. J Am Dent Assoc 2001, 132(Suppl):30S-35S.

8. Warnakulasuriya S: Effectiveness of tobacco counseling in the dental office. J Dent Educ 2002, 66:1079-1087.

9. Edwards D, Freeman T, Roche AM: Dentists' and dental hygienists' role in smoking cessation: an examination and comparison of current practice and barriers to service provision. Health Promot J Austr 2006, 17:145-51

10. Needleman I, Warnakulasuriya S, Sutherland G, Bornstein MM, Casals E, Dietrich1 T, Suvan J: Evaluation of tobacco use cessation (TUC) counselling in the dental office. Oral Health Prev Dent 2006, 4:27-47.

11. Carr AB, Ebbert JO: Interventions for tobacco cessation in the dental setting. A systematic review. Community Dent Health 2007, 24:70-74.

12. Gordon JS, Andrews JA, Crews KM, Payne TJ, Severson HH: The $5 A^{\prime}$ s vs $3 A^{\prime} s$ plus proactive quitline referral in private practice dental offices: preliminary results. Tob Control 2007, 16:285-288.

13. Rosseel JP, Jacobs JE, Hilberink SR, Maassen IM, Allard RH, Plasschaert AJ, Grol R: What determines the provision of smoking cessation advice and counselling by dental care teams? Br Dent J 2009, 206:376-377, E13; discussion.

14. Gonseth S, Abarca M, Madrid C, Cornuz J: A pilot study combining individual-based smoking cessation counseling, pharmacotherapy, and dental hygiene intervention. BMC Public Health 2010, 10:348.

15. Ohida T, Sakurai H, Mochizuki Y, Kamal AM, Takemura S, Minowa M, Kawahara K: Smoking prevalence and attitudes toward smoking among Japanese physicians. JAMA 2001, 285:2643-2648.

16. Naito T, Miyaki K, Naito M, Yoneda M, Suzuki N, Hirofuji T, Nakayama T: Parental smoking and smoking status of Japanese dental hygiene students: a pilot survey at a dental hygiene school in Japan. Int J Environ Res Public Health 2009, 6:321-328.

17. Nishina M, Taniguchi M, Murai K, Kojima K, Suzuki F, Kakizawa T: Nosmoking guidance for outpatients at Department of Internal Medicine, Tokyo Dental College, Suidobashi Hospital. Shikwa Gakuho 2009, 110:50-55[http://ir.tdc.ac.jp/irucaa/bitstream/10130/1204/1/110_50.pdf], in Japanese)

18. Matsui N, Tsuda T: The need for instruction on smoking cessation for outpatients compared with the actual guidance given by physicians in a general practice clinic. The Japanese Journal of Family Practice 2003, 10:44-49[http://jafm.org/journal/pdf/vol10no2/10_2_44matsui.pdf], (in Japanese).

19. U.S. Department of Health and Human Services: Clinical practice guideline, Treating tobacco use and dependence: 2008 Update. 2008, 35-36[http:// www.surgeongeneral.gov/tobacco/treating_tobacco_use08.pdf].

20. Binnie VI, McHugh S, Jenkins W, Borland W, Macpherson LM: A randomised controlled trial of a smoking cessation intervention delivered by dental hygienists: a feasibility study. BMC Oral Health 2007, 7:5. 
21. Martin LM, Bouquot JE, Wingo PA, Heath CW Jr: Cancer prevention in the dental practice: oral cancer screening and tobacco cessation advice. J Public Health Dent 1996, 56:336-340.

22. Johnson NW: The role of the dental team in tobacco cessation. Eur J Dent Educ 2004, 8:18-24.

23. Hu S, Pallonen U, McAlister AL, Howard B, Kaminski R, Stevenson G, Servos T: Knowing how to help tobacco users. Dentists' familiarity and compliance with the clinical practice guideline. J Am Dent Assoc 2006, 137:170-179.

24. Ibrahim H, Norkhafizah S: Attitudes and practices in smoking cessation counselling among dentists in Kelantan. Archives of Orofacial Sciences 2008, 3:11-16.

25. Ohmori M, Shizukuishi S, Hanioka T, Numabe $Y$, Aoyama H, Ishii M, Yoshie $\mathrm{H}$, Arai $\mathrm{T}$ : Smoking status and attitude to tobacco control policy among periodontists of the Japanese Scoiety of Periodontology. J Jpn Soc Periodontol 2009, 51:334-345, (in Japanese).

26. Kanesaka $Y$, Ooida $T$ : A report on perceptions regarding smoking among the members of the Japanese Medical Association. Japan Medical-Dental Association for Tobacco Control [http://www.nosmoke-med.org/PDF/ JDA2008.pdf], (in Japanese).

27. Hanioka T: Prevalence of smokers among dentists. Tobacco Control Medical-Dental Research Network [http://tobacco-control-research-net.jp/ action/dental.html], (in Japanese).

28. Nardina S, Bertoletti R, Rastelli V, Donner C: Influence of personal tobacco smoking on the clinical practice of Italian chest physicians. Eur Respir J 1998, 12:1450-1453.

29. Block D, Block L, Hutton S, Johnson K: Tobacco counselling practices of dentists compared to other health care providers in a midwestern region. J Dent Educ 1999, 63:821-826.

30. Sarna L, Brown J, Lillington L: Tobacco-control attitudes, adovocacy and smoking behaviours of oncology nurses. Oncol Nurs Forum 2000, 27:1519-1528

31. Dalia D, Palmer RM, Wilson RF: Management of smoking patients by specialist periodontists and hygienists in the United Kingdom. J Clin Periodontol 2007, 34:416-422.

32. Lancaster $T$, Stead L, Silagy $C$, Sowden A: Effectiveness of interventions to help people stop smoking: findings from the Cochrane Library. Br Med J 2000, 321:355-358.

doi:10.1186/1756-0500-3-329

Cite this article as: Saito et al:: Health professional's perceptions of and potential barriers to smoking cessation care: a survey study at a dental school hospital in Japan. BMC Research Notes 2010 3:329.

\section{Submit your next manuscript to BioMed Central and take full advantage of:}

- Convenient online submission

- Thorough peer review

- No space constraints or color figure charges

- Immediate publication on acceptance

- Inclusion in PubMed, CAS, Scopus and Google Scholar

- Research which is freely available for redistribution

Submit your manuscript at www.biomedcentral.com/submit
Biomed Central 